\title{
An Investigation on Dc Conduction in Multilayer Ceramic Capacitors
}

\author{
Non-Member ZHOU Yuanxiang (Akita University) \\ Member N. Yoshimura (Akita University)
}

Dc conduction through dielectric insulating materials has been the subject of intensive theoretical and experimental investigations in the study of dielectric material. It has been reported that the conduction current of multilayer ceramic capacitors at elevated temperature could be correlated to a thermally activated ionic transition process. In this paper, the conduction processes in $\mathrm{BaTiO}_{3}$ based multilayer ceramic capacitors (MLC) at room and elevated temperature were discussed from the dc conduction measurements. It was found that the dc conduction processes were different at these two temperatures.

At the elevated temperature, the conduction current could be caused by a thermally activated ionic transition process described. The hopping distance of dielectric material was calculated to be $60.3 \AA$, the carrier mobility of the electrical conduction was computed to be the order of $10^{-12} \mathrm{~cm}^{2} / \mathrm{V}$.s. It was estimated that the effective onset voltage of ionic conduction was higher than $6.9 \times 10^{4} \mathrm{~V} . \mathrm{cm}^{-1}$.

The conduction process at room temperature was also detail discussed. It was found that de conduction currents in MLC at room temperature were consistent with the values predicted by a theory based on the conventional thermally activated electronic injection process. It was obtained that the Ohmic field of MLC was lower than $55 \mathrm{kV} / \mathrm{cm}$. The resistivity of MLC was calculated to be $1.8 \times 10^{-13} \Omega \mathrm{cm}$ when subtracted the Ohmic current from the total measured current. It was estimated that the effective Schottky field was higher than $1.1 \times 10^{5} \mathrm{~V} . \mathrm{cm}^{-1}$. From these results, it is concluded that electronic conduction based on the Schottky mechanism may dominate the conduction current of MLC at room temperature.

It was presumed that the migration ion density in the bulk of dielectric material which changed with the temperature, was responsible for the different conduction processes.

Key words: Conduction current, Dielectric ceramics, Multilayer ceramic capacitor, Space charge

\section{Introduction}

Since 1943 , due to the discovery of the high permittivity $\varepsilon_{r}$ of ferroelectric barium titanate, ceramic materials based on this compound are utilized in the manufacture of ceramic capacitors. Development of multilayer ceramic capacitors becomes more important recently in the minimization of electronic instruments for the advantage of high frequency properties, small size and high capacitance by compared with the other sorts of capacitors. Due to the complicated fracturing process of the MLC and multiple thin ceramic layers between internal electrodes, defects such as impurities and structural anomalies are more easily formed in the dielectric material, which result in higher breakdown probability and lower reliability. Therefore, it is necessary to study the slectrical properties of the MLC before it's put into use

Dc conduction through dielectric insulating materials has been he subject of intensive theoretical and experimental investigations. Jnfortunately, only few papers have been published on electrical roperties of ceramic capacitors, especially on electrical properties if multilayer ceramic capacitors ${ }^{1-7)}$. It has been recognized that a thermally activated ionic conduction dominates the conduction current of multilayer ceramic capacitors at elevated temperature ${ }^{\text {") }}$. However, the conduction process of multilayer ceramic capacitors at room temperature has not been made clear so far

A unified model of dc leakage in $\mathrm{BaTiO}_{3}$ ceramic based on the Poole-Frenkel effect is proposed by Engene Loh $^{3)}$ to explain dc conduction process of ceramic capacitors. Though the measured slope of the $\log (\mathrm{I})-\mathrm{E}^{1 / 2}$ plot in the electrical conduction of thin $\mathrm{Ta}_{2} \mathrm{O}_{5}$ films corresponds well with the calculated Schottky plot, Peter L. Yong ${ }^{4)}$ suggested that the conduction in $\mathrm{Ta}_{2} \mathrm{O}_{5}$ films is dominated by Poole-Frenkel process. While based on the measured $\beta$, Pulfrey et al. ${ }^{5}$ have concluded that the conduction mechanism in $\mathrm{Ta}_{2} \mathrm{O}_{5}$ is via a Schottky process and, hence, is electrode limited. T. Mihara et al. ${ }^{6)}$ and P. Li et al. ${ }^{7)}$ have considered that according to different conditions, both the Schottky effect and Poole-Frenkel process may dominate the conduction current in PZT and $\mathrm{BaTiO}_{3}$ films. However, experimental results and their interpretations appeared to vary among different investigators, it is difficult to determine the conduction mechanism for dielectric materials.

In this paper, the conduction processes in $\mathrm{BaTiO}_{3}$ based multilayer ceramic capacitors (MLCs) at room and elevated 
temperature were both discussed from the dc conduction current measurements. Both long term leakage currents with and without polarity reversing at room and elevated temperature were measured, and comparison was made between these conduction results. However, the conduction process at room temperature was mainly discussed from the results of $\mathrm{dc}$ conduction current measurements, as well as the Schottky process and the PooleFrenkel mechanism were also introduced here to analyze the conduction process of MLC.
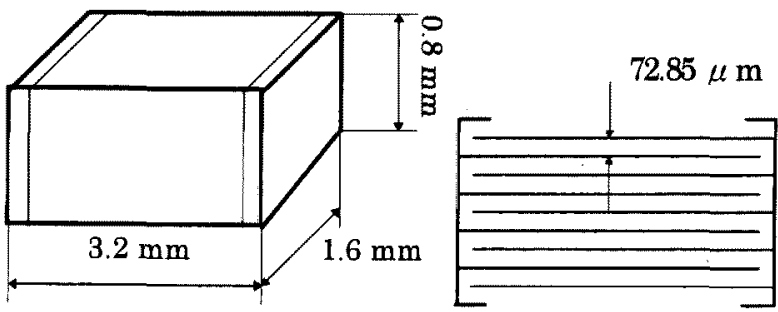

Fig. 1. The schematic structure of MLC.

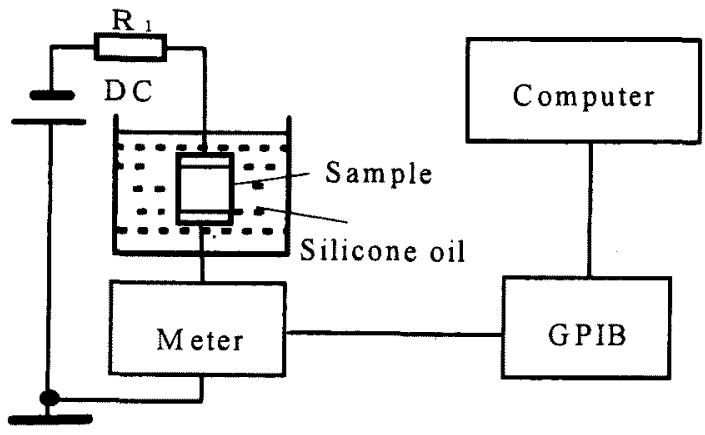

Fig. 2. Schematic diagram for dc current measurement

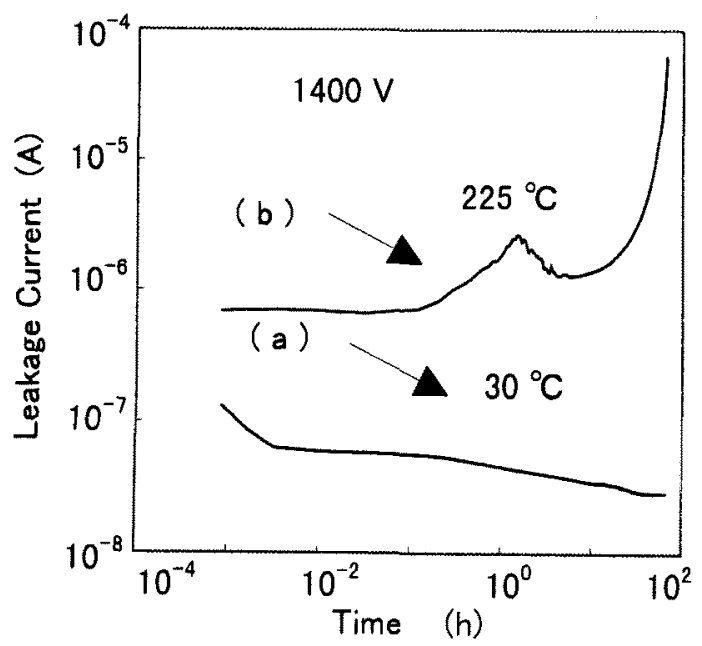

Fig. 3. Typical conduction current of MLC at (a) room, and (b) elevated temperature during stressing

\section{Experimental}

Fig. 1 shows the structure of MLC used in experiments. The MLC used in experiment consists of 8 layers with a schematic size of 3.2 $\times 1.6 \times 0.8 \mathrm{~mm}$, and a distance of $72.85 \mu \mathrm{m}$ between internal electrodes. The dielectric material in MLC is ceramic material based on $\mathrm{BaTiO}_{3}$ compound.

For the measurements of dc conduction, samples were put into silicone oil (1000 [CS]) to prevent the samples from flashover or discharge along the sample's surface. Samples were also put into oven ( HiTEC Yamato Fine Oven DF42) to control the experimental temperature. In order to avoid the influence of humidity, all samples were stored in a drying oven at $50{ }^{\circ} \mathrm{C}$ before any subsequent measurement. As shown in Fig. 2, dc conduction current was measured by computer using GP-IB connected to an ADVANTEST R8340A Ultra High Resistance Meter, and a 2 min value was taken as a datum.

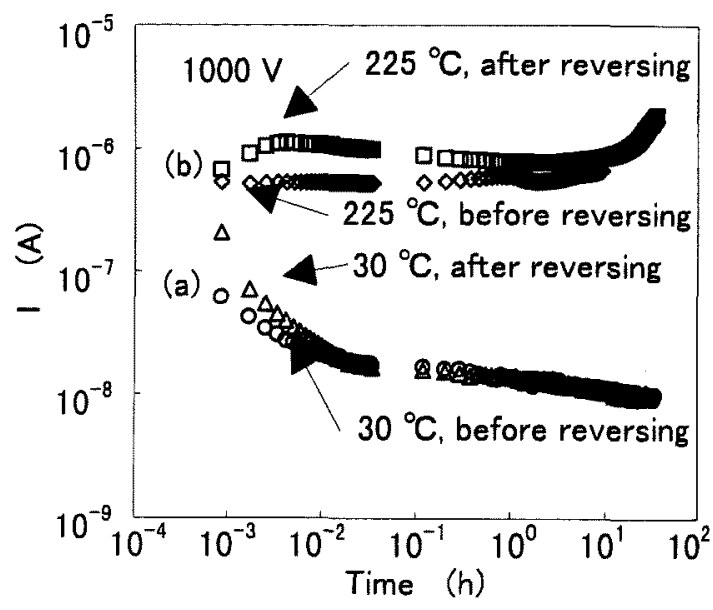

Fig. 4. The conduction currents before and after polarity reversing at (a) room, and (b) elevated temperature.

\section{Results and discussion}

$<3.1>$ Behavior of dc conduction Physical properties of dielectric materials will change with temperature. In this paper, two temperatures which were $30{ }^{\circ} \mathrm{C}$ and $225{ }^{\circ} \mathrm{C}$, were selected to discuss the conduction process at room temperature and elevated temperature in MLC, respectively. Fig. 3 shows a typical change in dc charging current curves as a function of the voltage application time observed at room temperature $\left(30^{\circ} \mathrm{C}\right)$ and elevated temperature ( $225{ }^{\circ} \mathrm{C}$ ) for MLC. Generally, the time-dependent leakage current will show a big current at the moment of applying voltage and then decreases with time, finally tends to a steady state due to the absorption of charges in dielectric material. However, in the present work, the curves in Fig. 3 are of special properties. At elevated temperature, the leakage current does not show the 
mentioned characteristics. No obvious absorption current is observed. The leakage current increases with time and shows a peak during the period of current increasing, finally leads to dc breakdown of MLC. On the other hand, a transient absorption current can be seen before the current reaches to a relative steady state at room temperature. Thereafter, the current decreases very slowly for a long time about 80 hours before it tends to a constant again.

<3.1. Space charge accumulation The abnormal current appears at elevated temperature is due to ionic conduction of MLC as described ${ }^{1)}$. It can be further confirmed by measuring the polarity reversed conduction current as shown in Fig. 4 . It has been described that there are peak currents appeared in ionic conduction dominant currents when applying polarity reversed voltage to samples ${ }^{1,8,9)}$ as shown in Fig. 4 at elevated temperature.
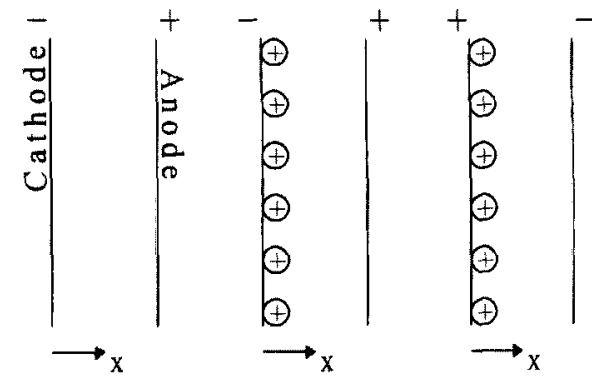

$$
\begin{array}{ccc}
\mathrm{t}=0 & \begin{array}{c}
\text { Charges } \\
\text { formation }
\end{array} & \begin{array}{c}
\text { Polarity } \\
\text { reversed }
\end{array} \\
(\mathrm{a}) & (\mathrm{b}) & (\mathrm{c})
\end{array}
$$

Fig. 5. The formation of space charges described.

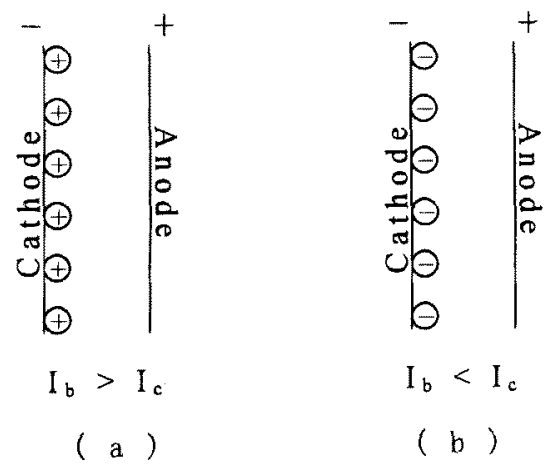

Fig. 6. The relationship between conduction current and space charge formation. $I_{b}$ is bulk current, $I_{c}$ is electrode injection current.

According to Miyairi et al ${ }^{97}$ and Yoshimura ${ }^{1)}$, like in PET and in the other sort of MLC, the internal distribution field in dielectric material is influenced by the hetero space charge which accumulates near the cathode. The abnormal current measured here at elevated temperature can be explained by using the space charge accumulation model shown in Fig. 5 . When the sample was applied with dc voltage, the positive charges in bulk transported along the field to cathode, then accumulated near the cathode. After the charge accumulating, the distribution field near the cathode was increased, causing a suddenly injection of numerous electrons from cathode into dielectric material, resulting in the neutralization of the accumulated charges near the cathode which led to the peak current. After a certain number of charges was neutralized, the large discharging current disappeared, only conduction current flowed in the bulk. From Figs. 3 and 4 , it can be seen that the current at elevated temperature is well correlated with this picture.

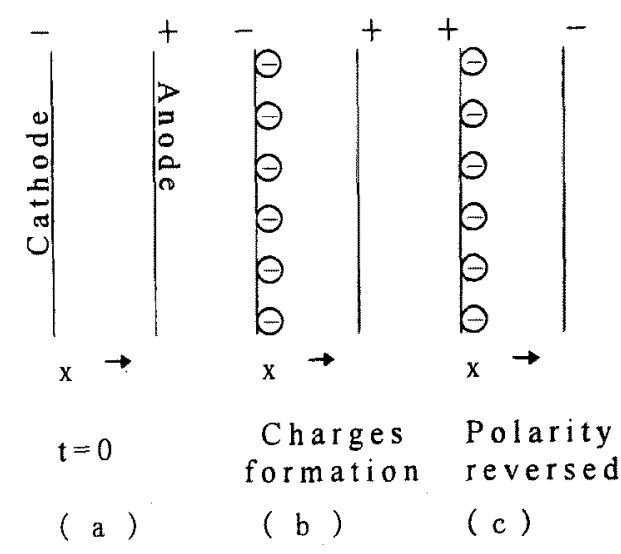

Fig. 7. The formation of space charge at room temperature.

However, the model depicted in Fig. 5 is not correlated with the conduction picture at room temperature. The different conduction behavior at different temperature indicates that the conduction process of MLC at room temperature is different to the one at elevated temperature. In fact, if both the electrons injected from the electrode and the ions transported in the bulk were considered to be in existence simultaneously, the model shown in Fig. 5 can also be well explained. In other words, as shown in Fig. 6, the space charge accumulation is dependent on the relationship between the bulk current $I_{b}$ and electrode injection current $\mathrm{I}_{c}{ }^{10}$ ) $\mathrm{I}_{\mathrm{b}}$ is caused by migration ions in the bulk, and $I_{c}$ is caused by electrons injected from cathode. When $I_{b}>I_{c}$ as shown in Fig. 6 ( a ), positive charges will be transported and accumulated near the cathode due to the migration of the ions, resulting in hetero charge formation as shown in Fig. 5 (b). When $I_{b}<I_{c}$ as shown in Fig. $6(b)$, electrons will accumulate near the cathode due to the injection of electrons from cathode, resulting in homo charge formation as shown in Fig. 7 (b). During the period of the charge accumulating, both the injection of electrons from cathode and the transition of ions in bulk are in existence simultaneously.

The ionic transition and electronic conduction in dielectric 
materials are thermally excited. According to Ieda, Shinohara, and Miyairi ${ }^{8,9)}$, the ionic conduction current is greatly influenced by testing temperature. When temperature is low, the density of ions distributing in bulk is low, and there are hardly migration ions in existence. The density of migration ions will increase with temperature, causing an increasing ionic conduction current. At the same time, the electrons injected from cathode will also increase with temperature. It can be assumed that the increase speed of migration ions with temperature is different from that of injection number of electrons, resulting in different relationship between $I_{b}$ and $I_{c}$.

Therefore, at elevated temperature, the migration ion density in bulk is high and the speed of ion migration is higher than the electron number injected from cathode, setting up an $I_{b}>I_{c}$ relationship between $I_{b}$ and $I_{c}$, resulting in an ionic transition dominant dc conduction current at elevated temperature as shown in Figs. 3 and 4. This well correlates with the model described in Fig. 5. On the other hand, at room temperature, the migration ion density in bulk is low and the speed of ion migration is lower than that of the electron number injected from cathode under do stressing, setting up an $I_{b}<I_{c}$ relationship between $I_{b}$ and $I_{c}$ resulting in an electronic emission dominant $\mathrm{dc}$ conduction current at room temperature as shown in Figs. 3 and 4

Table 1. Dc prestressing effect on dc breakdown of MLC at room temperature: prestressing time $20 \mathrm{~min}$, field 165 $\mathrm{kV} / \mathrm{cm}$, temperature $30^{\circ} \mathrm{C}$.

\begin{tabular}{|l|c|}
\hline $\begin{array}{l}\text { Prestressing conditions } \\
\text { and applying voltages }\end{array}$ & $\begin{array}{c}\text { Dc electric strength } \\
(\mathrm{kV} / \mathrm{cm})\end{array}$ \\
\hline Without prestressing ang and & 371 \\
\hline $\begin{array}{l}\text { After prestressing, and } \\
\text { applying dc voltage without } \\
\text { polarity reversing }\end{array}$ & 459 \\
\hline $\begin{array}{l}\text { After prestressing, and } \\
\text { applying dc voltage with } \\
\text { polarity reversing }\end{array}$ & 250 \\
\hline
\end{tabular}

\section{<3.1.2> Electronic injection Furthermore, as shown} in Table 1, the injection and accumulation of electrons near cathode at room temperature can be well explained by dc prestressing effect on dc breakdown measurements. After prestressing by a dc field of $165 \mathrm{kV} / \mathrm{cm}$ for $20 \mathrm{~min}$ at room temperature, the dc electric strengths of MLC with and without polarity reversing were measured, as well as the dc electric strength of sample without prestressing was measured. By making comparison with the dc electric strength of the sample without prestressing, it was found that after prestressing, the dc electric strengths of samples obviously increased and decreased when applying $\mathrm{dc}$ voltage without and with polarity reversing, respectively. These prestressing effects on dc breakdown have been intensively investigated by lots of investigators ${ }^{11,12)}$, it indicates that there are electrons injected and accumulated near the cathode during prestressing as shown in Fig. 7. However, it is clear that the picture depicted in Fig. 7 shows the space charge accumulation in MLC at room temperature.

Therefore, at room temperature, electrons are injected and accumulated near the cathode. At the moment of voltage applying to the sample, a transient absorption current will occur and quickly reaches to a steady state before the charges accumulating. After the space charges accumulating as shown in Fig. 7 (b), the barrier between cathode and dielectric material becomes higher, resulting in a resistance of the injection of electrons. With the increasing space charges, the injection of electrons becomes more difficult, finally tends to a steady state in which only the conduction current exists. Electrons injected into dielectric material can be quickly extracted by applying with a polarity reversed field ${ }^{13)}$. At the moment of polarity reversing, as shown in Fig. 7 (c), a transient extraction current of the injected electrons near anode accompanied with an absorption current will occur and quickly tends to a steady state. Then the conduction current shows a similar process as the one without polarity reversed. The injected electrons hardly show affect on the conduction process after polarity reversed for a certain period. Therefore, according to Fig. 7 , the conduction current at room temperature shown in Figs. 3 and 4 can be well explained

The Schottky emission and the Poole-Frenkel mechanism have often been assumed as alternative mechanisms for explaining experimental results in the case when the current density exponentially increases with the square root of applying field

If conduction in the case of room temperature is mainly governed by bulk processes in the material, homocharge accumulation hardly occur. When trapped electrons are thermally excited into the conduction band, space charge with different polarity as the electrode (heterocharges) should be observed after prestressing. In the present experiment, as shown in Table 1, there is no hetero effect can be seen in dc breakdown after prestressing. On the other hand, electrode limited conduction current easily forms homo space charges and shows homo effect in dc breakdown. Therefore, of the two possibilities, the Poole-Frenkel process can be excluded from the mechanism of dc conduction. As a conclusion of the present Section, it can be said that the conduction process in MLC at room temperature is mainly due to electrons emission from cathode as will be discussed later.

\section{$<3.2>$ Dc Conduction at room Fig. 8 shows the} change in dc charging current curves as a function of the voltage application time observed at $30{ }^{\circ} \mathrm{C}$. All the curves seem to reach their respective steady states after $120 \mathrm{~s}$. Therefore, 2 min values were chosen as data to discuss the conduction behavior as a function of the applied field. Fig. 9 shows this dependence. The conduction current increases as the field and temperature increase. 
This indicates that the current is dominated by a thermally activated process.

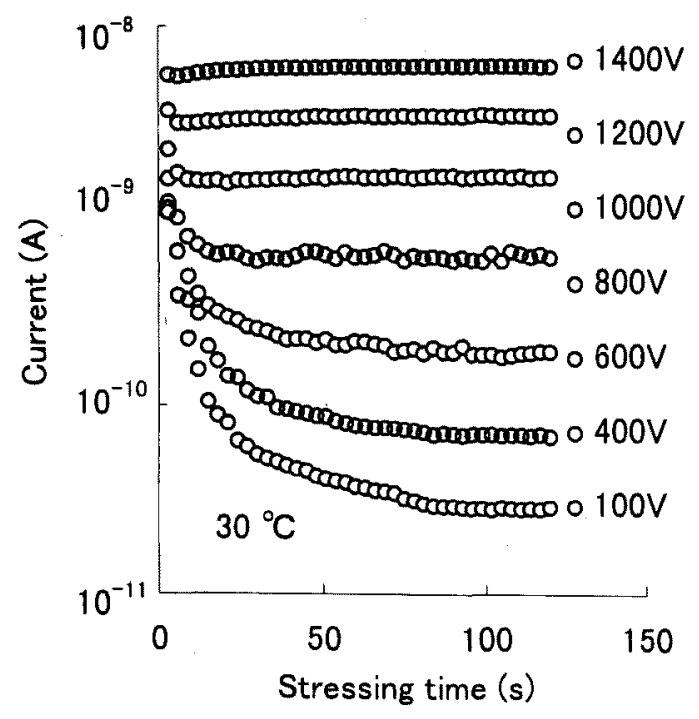

Fig. 8. Decay profiles of the charging current observed at $30{ }^{\circ} \mathrm{C}$ with various applied voltages.

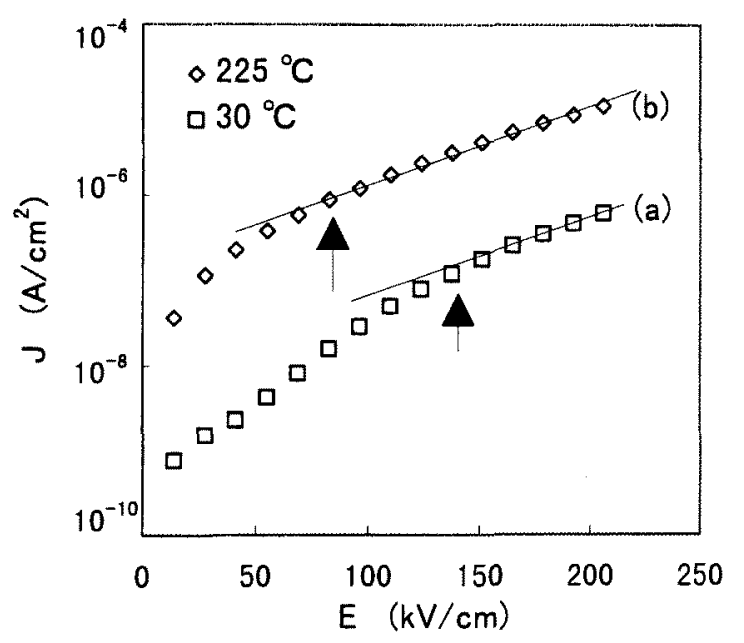

Fig. 9. Typical curves on $\log (\mathrm{J})$ vs. E plot at (a) room, and (b) elevated temperature

Generally, the conduction mechanism is related with the valtage range over which the current of this conduction mechanism predominates. After lots of theoretical analyzing and calculating, according to the voltage range over which the thermionic current predominates, Simmons ${ }^{15}$ ) has reported that the thermionic current predominating conduction will change to tunnel current at temperature lower than $300 \mathrm{~K}$. Shimada et al. ${ }^{16)}$ have introduced this conception to make comparison between the conduction current of two kinds of capacitors, they concluded that one of these capacitors is more strongly governed by the Poole-Frenkel mechanism is due to the lower onset voltage of Poole-Frenkel plot. The obvious difference between the onset voltage of straight lines indicated by arrows in Fig. 9 implies that an ionic flow strongly predominates the conduction current at elevated temperature, while at room temperature, it is negligible. That is well correlated with the picture mentioned

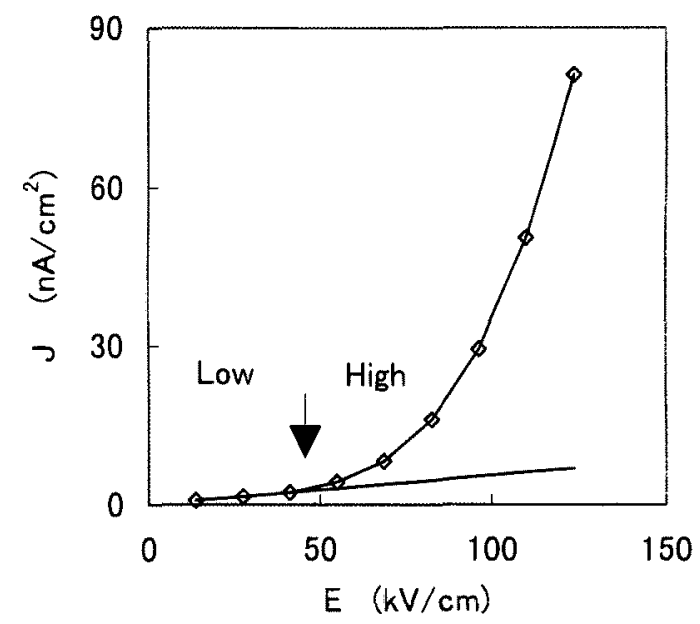

Fig. 10. Typical Ohmic behavior of a MLC at low stress. $\rho \sim 0.18 \times 10^{14} \Omega \mathrm{cm}$.

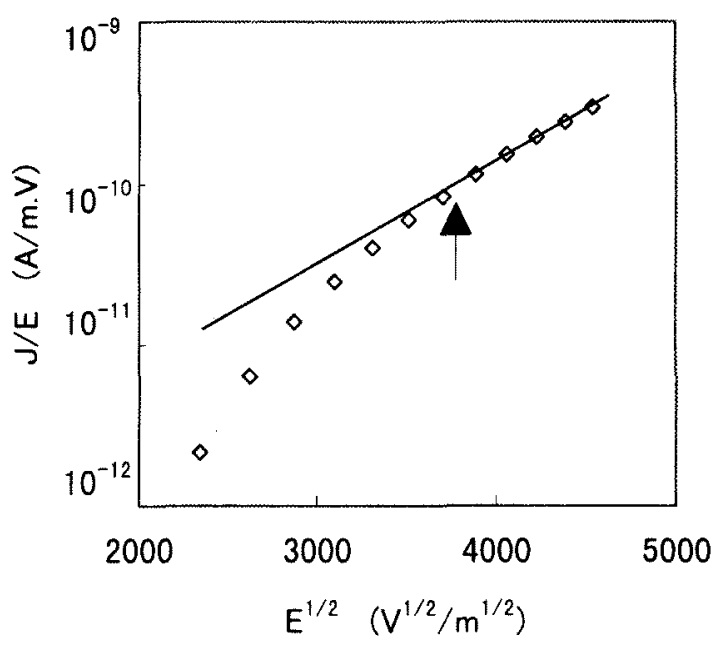

Fig. 11, Typical $\log (J / E)$ vs. $E^{1 / 2}$ plot for MLC at room temperature.

3.2.1> Ohmic behavior In fact, the current-voltage (I-V) characteristics at low fields exhibits an Ohmic behavior (Fig. 10). If we assume that separate conduction mechanisms are operative in the low and high field regions, then the Ohmic current should be subtracted from the total measured current in discussing conduction at high fields. Indeed, a straight line can be fitted to the data points (Fig. 11 and Fig. 12). From the experimental results, two regions with different conduction characteristics have been identified. In the low field region $(\mathrm{E}<55 \mathrm{kV} / \mathrm{cm})$ the data can best 
fitted in terms of an Ohmic mechanism (Fig. 10). Arrow in Fig. 10 indicates the point of approximate end field of the Ohmic behavior on curve. The Ohmic region voltage below $55 \mathrm{kV} / \mathrm{cm}$ is almost the same as $50 \mathrm{kV} / \mathrm{cm}$ of the Ohmic voltage region reported ${ }^{2)}$. The resistivity in this region is about $0.18 \times 10^{14} \Omega \mathrm{cm}$.

The Poole-Frenkel and the Schottky effects can be thermally activated. The high field conduction data is analyzed in terms of the two models. In all subsequent data analysis, the Ohmic contribution to the measured currents has been subtracted.

$<3.2$.2 The Poole-Frenkel process If the conduction process is controlled by the Poole-Frenkel effect, the conductivity is expressed by the following equations ${ }^{10 \text { ) }}$

$$
\begin{aligned}
& \sigma=\sigma_{0} \exp \left[\frac{\beta_{P F} E^{1 / 2}}{\kappa T}\right] \\
& \beta_{P F}=\left[\frac{e^{3}}{\pi \varepsilon_{0} \varepsilon_{r}}\right]^{1 / 2}
\end{aligned}
$$

Here $\sigma, \beta_{P F}, \varepsilon_{0}$, and $\varepsilon_{r}$ are the conductivity, Poole-Frenkel coefficient, permittivity of free space, and relative permittivity, respectively. The value of permittivity is calculated from the slope of the Poole-Frenkel plot $\left(\log \sigma\right.$ vs. $\left.E^{1 / 2}\right)$ for the measured data shown in Fig. 11, and the result is $\varepsilon_{r}=8611$. Arrow in figure indicates the point of approximate onset field of the linear behavior on curve. The onset field is about $137 \mathrm{kV} / \mathrm{cm}$.

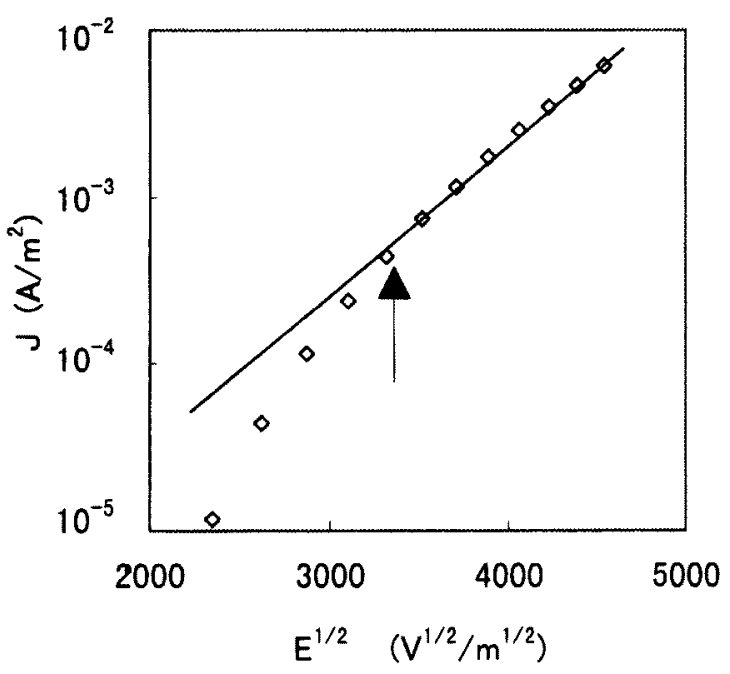

Fig. 12. Typical $\log (J)$ vs. $E^{1 / 2}$ plot of a MLC at room temperature.

<3.2.3> Schottky process If the Schottky effect is dominant in the conduction process, the current density would be given by ${ }^{10)}$

$$
\begin{aligned}
& J=A T^{2} \exp \left[-\frac{\phi}{\kappa T}\right] \exp \left[\frac{\beta_{S} E^{1 / 2}}{\kappa T}\right] \\
& \beta_{S}=\left[\frac{e^{3}}{4 \pi \varepsilon_{0} \varepsilon_{r}}\right]^{1 / 2}
\end{aligned}
$$

Here $\mathrm{A}$ is the Richardson-Dushman constant, $\beta_{S}$ is the Schottky coefficient, and $\Phi$ is the barrier height between the electrode and dielectric ceramic. The Schottky plot $\left(\log \mathrm{J}\right.$ vs. $\left.\mathrm{E}^{1 / 2}\right)$ is shown in Fig. 12. Arrow in figure indicates the point of approximate onset field of the linear behavior on curve. The permittivity value calculated from the slope is 2653 . The onset field is about $110 \mathrm{kV} / \mathrm{cm}$. From the slope of $\log \left(\mathrm{J} / \mathrm{T}^{2}\right)$ vs. $1 / \mathrm{T}$ plot, the activation energy is calculated to be $0.20 \mathrm{eV}$

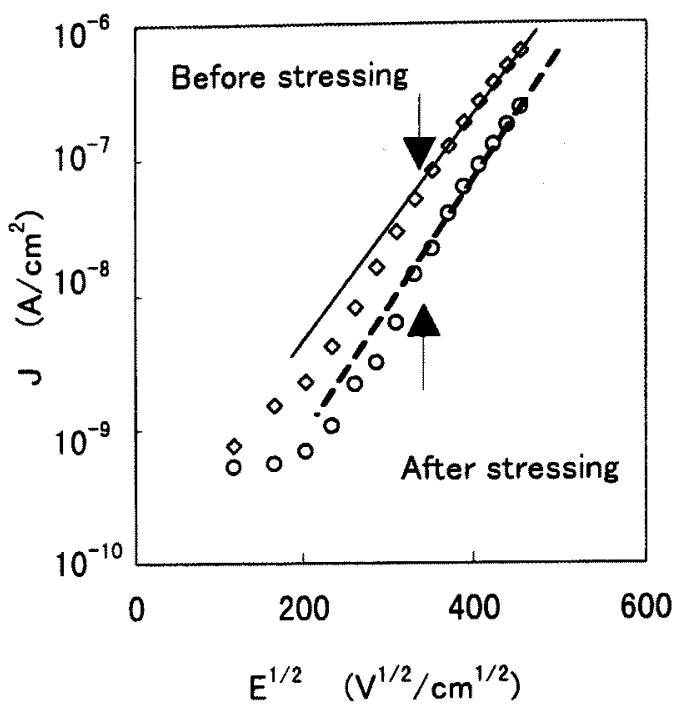

Fig. 13. Typical J-V curves on $\log (J)$ vs. $E^{1 / 2}$ plot. No Ohmic contribution is subtracted. (a) before prestressing, (b) after prestressing at $30{ }^{\circ} \mathrm{C}$ at $1400 \mathrm{~V}$ for $120 \mathrm{~h}$.

\section{<3.2.4> Predominant conduction process at room} temperature As described above, the electronic conduction process dominates the dc conduction current at room temperature. If comparison is made between the Poole-Frenkel process and the Schottky process as shown in Figs. 11 and 12, it is found that the onset voltage for the Schottky plot is $110 \mathrm{kV} / \mathrm{cm}$ while for the Poole-Frenkel plot is $137 \mathrm{kV} / \mathrm{cm}$. This indicates that the conduction current appears to be more strongly governed by the Schottky mechanism. However, if comparison was further made with the real permittivity value of 2900 measured at room temperature ( $\left.1 \mathrm{kHz}, 1 \mathrm{~V}_{r m s}\right)$, the permittivity value 8611 calculated from the Poole-Frenkel plot $\left(\log (\mathrm{J} / \mathrm{E})-\mathrm{E}^{1 / 2}\right)$ is far away than the permittivity value 2653 calculated from the Schottky plot $(\log (\mathrm{J})$ $\mathrm{E}^{1 / 2}$ ). The value of activation energy $0.20 \mathrm{eV}$ is also appropriate if by making comparison with the values reported such as $0.3 \mathrm{eV}$ for capacitor which consist of $\mathrm{BaTiO}_{3}$ ceramic ${ }^{3)}, 0.58 \mathrm{eV}$ for sol-gel PZT thin-film capacitor ${ }^{6)}$, and $0.2 \mathrm{eV}$ for ceramic material consists of $\mathrm{SrTiO}_{3}-\mathrm{MgTiO}_{3}-\mathrm{Bi}_{2} \mathrm{O}_{3} \cdot \mathrm{TiO}_{2}{ }^{14)}$. For these cases, it can be concluded that the conduction current of MLC is dominated by the Schottky mechanism rather than by the Poole-Frenkel mechanism. 
This indicates that the conduction in MLC is predominated by electrode-limited processes but not by bulk-controlled processes as mentioned above. Moreover, the onset voltage for the Schottky plot is $110 \mathrm{kV} / \mathrm{cm}$ which is similar to the value described ${ }^{3)}$.

As a conclusion of the present Section, it can be said that the dc conduction current in MLC is mainly due to the conduction process of thermally electronic emission based on the Schottky mechanism. In the case after prestressing at $1400 \mathrm{~V}$ about $120 \mathrm{~h}$, as shown in Fig. 13, dc voltage with the same polarity has been applied to measure the J-V curves. After prestressing, electrons are injected and accumulated near the cathode, cause a higher barrier between cathode and dielectric material. The accumulating space charges show a resistance effect on the injection of electrons. Due to these space charges, the injection of electrons becomes more difficult, resulting in a decreasing conduction current of dielectric material. When applying voltage on MLC after prestressing, the conduction current is decreased by the resistant effect of the space charge accumulated during prestressing. And it is evident that the current after prestressing is less than that without prestressing as shown in Fig. 13. Furthermore, this improves that the electronic conduction current is limited by the trapped electrons injected from cathode. In other words, electrons are trapped in the interface of electrode and dielectric material during prestressing, which raise the barrier of the interface, causing a resistance of electronic injection, resulting in the decrease of conduction current. These results are well correlated with the case mentioned in Fig. 7. For a further study of the effect of space charge on dc conduction, more experiments must be carried out to find out the defects which influence the conduction behavior of MLC. However, the oxygen vacancies, metal vacancies, and interstitial metals may act as possible roles of traps in MLC.

\section{$<3.3>$ De Conduction at elevated temperature}

Because the conduction currents are more easy to reach their respective steady states at elevated temperature than at room temperature, the $2 \mathrm{~min}$ values mentioned above are reasonably chosen as data to discuss the conduction behavior as a function of the applied field at elevated temperature

The relationship between current density $\mathrm{J}$ and applying field $\mathrm{E}$ at elevated temperature is also shown in Fig. 9. From the linear behavior of $\log (\mathrm{J})$ vs. E plot, and confirming the results discussed above, it can be concluded that the ionic current dominates the conduction current of MLC at elevated temperature. Assuming that ions are transported by thermally assisted hopping, the current density is theoretically expressed by the following equation ${ }^{10)}$

$$
J \approx \operatorname{enav} \exp \left(\frac{e E a}{2 \kappa T}\right) \exp \left(-\frac{U}{\kappa T}\right)
$$

where $e$ is the electron charge, $n$ is the carrier density, $a$ is the hopping distance, $v$ is the attempt-to-escape frequency, $U$ is the activation energy, $\kappa$ is the Bolztmann constant, $T$ is the absolute temperature, and $E$ is the electrical field. The hopping distance is calculated to be $60.3 \AA$. From Fig. 3, if the time when the current reached to it's peak value was considered as the time for ions to migrate from one electrode to another ${ }^{1,8)}$, then according to

$$
\mu=\frac{v}{E}=\frac{l / t_{p}}{V / l}
$$

where $\mu$ is the ion mobility, $v$ is the speed of ion migration, $l$ is the distance between internal electrodes, $V$ is applying voltage, the mobility of dielectric material is computed to be $7.3 \times 10^{-12}$ $\mathrm{cm}^{2} /$ V.s

Arrow in Fig. 9 indicates the point of approximate onset field of the linear behavior on curve. The onset field is about $69 \mathrm{kV} / \mathrm{cm}$. The hopping distance value calculated from the slope is similar to the value of $400 \AA$ in other type of MLC ") and values in polymers ${ }^{10}$. The mobility value is also appropriate if comparison is made with the values reported for other materials such as $10^{-11}$ $\sim 10^{-12} \mathrm{~cm}^{2} / V$.s for the other type of MLC ${ }^{1)}$ and $10^{-8} \mathrm{~cm}^{2} / \mathrm{V}$.s for polymers ${ }^{10}$. The conductivity can not be calculated here due to the strongly ionic conduction current at elevated temperature and instrumental problems.

\section{Conclusions}

Both the dc conduction of MLC at room ( $30{ }^{\circ} \mathrm{C}$ ) and elevated temperature ( $225{ }^{\circ} \mathrm{C}$ ) are theoretically and experimentally discussed. And it can be described as follows.

(1) These authors can see no way in which the usual bulkcontrolled processes can be made to explain the observed experimental characteristics at room temperature. It is evident that charges are injected and accumulated near the electrode/dielectric interface of MLC at room temperature, while ions are transported and accumulated near the cathode at elevated temperature

(2) From the results of dc conduction current measurements, thermally activated electronic injection process is considered to be responsible for $\mathrm{dc}$ conduction in $\mathrm{MLC}$ at room temperature, where the effective Schottky field is higher than $1.1 \times 10^{5} \mathrm{kV} / \mathrm{cm}$. The Ohmic property of MLC exists in lower field region below 55 $\mathrm{kV} / \mathrm{cm}$. The resistivity of MLC was calculated to be $1.8 \times 10^{13} \Omega \mathrm{cm}$ when subtracted the Ohmic current from the total measured current. The conduction mechanism in MLC appears to be via a Schottky process and, hence, is electrode limited.

(3) Thermally activated ionic transition process is considered to dominate the $d c$ conduction current at elevated temperature. The obtained hopping distance is $60.3 \AA$, the ionic mobility of dielectric material is calculated to be $7.3 \times 10^{-12} \mathrm{~cm}^{2} / \mathrm{V}$.s.

(4) The dc conduction current can be dominated by different conduction process due to the change of migration ion density in bulk which changes with temperature. It is considered that the relationship between the space charge accumulation and conduction process discussed here will be useful to study the electrical properties of dielectric materials. 
(5) More detail experiments must be carried out to find out the defects which influence the accumulation of space charge. And more systematic study on the conduction process is expected.

\section{Acknowledgments}

Thanks to Dr. M. Suzuki, Dr. WANG Xingsheng, Dr. I. Mitobe, and Mr. T. Sato of Akita University for providing advice and support in this work. The authors are also grateful to $\mathrm{Mr}, \mathrm{T}$. Ogasawara and TDK Co. for experimental materials.

(Manuscript received Apr. 24, 1998; revised Jun. 17, 1998)

\section{References}

(1) N. Yoshimura, A. Ito, J. Ideguchi, and T. Ogasawara, "Electrical Conduction of Multilayer Ceramic Capacitor", Trans. IEE Japan, Vol. 111-A, No. 6, pp. 560-563, 1991 (in Japanese).

(2) G. M. Rao, and S. B. Krupanidhi, "Study of electrical properties of pulsed excimer Laser Deposited Strontium Titanate Films", J. Appl. Phys. 75 (1994) 2604.

(3) E: Loh, "A Model of Dc Leakage in Ceramic Capacitors", J. Appl. Phys. Vol. 53, No. 9, pp. 6229-6235, 1982.

(4) Peter L. Young, "Dc Electrical Conduction in Thin $\mathrm{Ta}_{2} \mathrm{O}_{5}$ Films. I .”, J. Appl. Phys., Vol. 47, No. 1, pp. 235-241, 1976.

(5) D. L. Pulfrey, P. S. Wilcox, and L. Young, "Dielectric Properties of $\mathrm{Ta}_{2} \mathrm{O}_{5}$ Thin Films", J. Appl. Phys. 40, pp. 3891-3898, 1969.

(6) T. Mihara, and H. Watanabe, "Electronic Conduction Characteristics of Sol-Gel Ferroelectric $\mathrm{Pb}\left(\mathrm{Zr}_{0.4} \mathrm{Ti}_{0.6}\right) \mathrm{O}_{3}$ Thin-Film Capacitors: Part I ", Jpn. J. Appl. Phys., Part 1, Vol. 34, pp. 5664-5673, 1995.

(7) P. Li and T. M. Lu, "Conduction Mechanisms in $\mathrm{BaTiO}_{3}$ thin films", Phys. Rev. B, Vol. 43, No. 17, pp. 14261-14264, 1991.

(8) M. Ieda, U. Shinohara, "On the Absorption Phenomena in the Plasticized Polyvinyl Chloride Resin by Direct Current Voltage", Trans. IEE Japan, Vol. 79, No. 850, pp. 843-850, 1959.

(9) K. Miyairi, M. Ieda, "Nonlinear Electrical Conduction and Space Charge Effect in Polyethylene Terephthalate", Trans. IEE Japan, Vol. 96-A, No. 1, pp. 25-30, 1976.

(10) M. Ieda, "Theory of Dielectric Phenomenon", Communication \& education group of electrical science committee, 1973 (in Japanese)

(11) M. Nagao, Y. Mizuuno and M. Kosaki, "Electrical Breakdown of Polar Polymeric Films in Cryogenic Temperature Region", Trans. IEE Japan, Vol. 112-A, No.3, pp. 196-201, 1992 (in Japanese).

(12) N. Yoshimura and F. Noto, "Effects of Dc Prestressing on
Tree Initiation in Polyethylene with and without NeedleShaped Void", Trans. IEEE, Vol. EI-19, No. 2, pp. 135-140, 1984.

(13) N. Yoshimura, Y. Koike, Sh. Fujita and F. Noto, "Space Charge Effect Associated with Tree-Initiation in Polyethylene under Square Pulse Conditions", Trans. IEE Japan, Vol. 104, No. 8, pp. 457-463, 1984.

(14) N. Yoshimura, A. Ito, J. Funaki, and T. Ogasawara, "Effect of Pore on Electrical Conduction and Dielectric Breakdown for Dielectric Ceramics", Trans. IEE of Japan, Vol. 108-A, No. 4, pp. 155-161, 1988 (in Japanese).

(15) John G. Simmons, "Potential Barriers and Emission-Limited Current Flow Between Closely Spaced Parallel Metal Electrodes", J. Appl. Phys., Vol. 35, No. 8, pp. 2472-2481, 1964.

(16) Y. Shimada et al., "Time-Dependent Leakage Current Behavior of Integrated $\mathrm{Ba}_{0.7} \mathrm{Sr}_{0.3} \mathrm{TiO}_{3}$ Thin Film Capacitors during Stressing", Jpn. J. Appl. Phys., Vol. 35, No. 9B, pp. 4919-4924, 1996

Zhou Yuanxiang (Non-member) He was born in Dec., 1966.

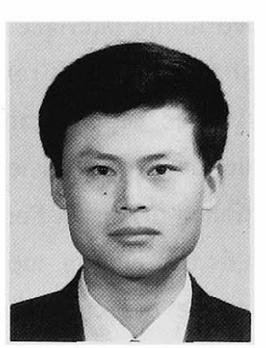

$\mathrm{He}$ received his B.E. degree in electrical engineering from Tsinghua University, China in 1988. He received his M.E. degree in electrical engineering from the Electrical Power Research Institute (EPRI) of China in 1991. He then joined EPRI to engage in electrical engineering. He shared a senior engineer treatment, and became the Head of the Group of Diagnosis and On-line Monitoring of Electrical Equipment of EPRI, China in 1995. He became a Doctor Course Student of Akita University in 1996. Now he is interested in dielectric materials, especially in ceramics.

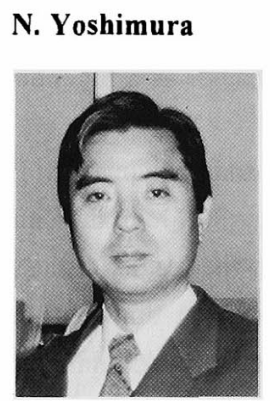

(Member) He was born in Nov., 1943. He received his B. E. and $\mathrm{M}$. E. degrees in electrical engineering from Akita University in 1967 and 1969 respectively, and Dr. of engineering from Nagoya University in 1975. He joined Akita University in 1969, and was the Dean of Mining College, Akita University from 1995 to 1998 . He has been a professor since 1983, a consulting professor of Xi'an Jiao Tong University of China since 1998, and the first Dean of the Faculty of Engineering and Resource Science, Akita University since 1998. He was a research Associate at Clarkson College of Technology, New York from Jan., 1978 to Sept., 1979. His research interests include dielectric materials of polymers, ceramics and semiconductive ceramics. 